JOURNAL OF

FUNCTION SPACES AND APPLICATIONS

Volume 5, Number 3 (2007), 213-230 (c) 2007, Scientific Horizon

http://www.jfsa.net

\title{
Bloch spaces of holomorphic functions in the polydisk
}

\section{Anahit Harutyunyan*}

(Communicated by Jürgan Appell)

2000 Mathematics Subject Classification. 32A18, 46E15.

Keywords and phrases. Weighted Bloch spaces, polydisk, projection, diagonal mapping.

\footnotetext{
Abstract. This work is an introduction to anisotropic spaces of holomorphic functions, which have $\omega$-weight and are generalizations of Bloch spaces to a polydisc. We prove that these classes form an algebra and are invariant with respect to monomial multiplication. Some theorems on projection and diagonal mapping are proved. We establish a description of $\left(A^{p}(\omega)\right)^{*}\left(\right.$ or $\left.\left(H^{p}(\omega)\right)^{*}\right)$ via the Bloch classes for all $0<p \leq 1$.
}

\section{Introduction}

The aim of this paper is to extend the Bloch spaces to a polydisk so that the well-known properties of the Bloch spaces of one variable remain true. Moreover, our generalization gives results which are new also for function of one variable. We are interested, for instance, in theorems on projection and the description of $\left(A^{p}(\alpha)\right)^{*}$ via Bloch spaces. One can consider other generalisations of the Bloch space for polydisk (see for example [1]).

${ }^{*}$ Supported by the Deutscher Akademischer Austauschdienst (DAAD). 
In the paper of F. Shamoyan [2], the spaces $A^{p}(\alpha)$ have been generalized and investigated as $H^{p}(\omega)$ spaces in a polydisk. The Bloch spaces play the same role for $A^{p}(\alpha)$ as the BMOA spaces for the Hardy theory. Hence, it is very important to generalize the $\omega$ weighted Bloch spaces to a polydisk.

In Section 1, such classes are defined and an auxiliary lemma is proved. In Section 2, some theorems describing the properties of the introduced Bloch spaces are proved. In particular, we prove that they are invariant with respect to monomial multiplication and in the some case they are a Lipschitz class. In Section 3, we consider bounded operators in these classes and diagonal mappings. Section 4 is devoted to a description of $\left(A^{p}(\omega)\right)^{*}$ via the introduced Bloch spaces.

\section{Preliminaries and basic constructions}

Let

$$
U^{n}=\left\{z=\left(z_{1}, \ldots, z_{n}\right) \in C^{n},\left|z_{j}\right|<1,1 \leq j \leq n\right\}
$$

be the unit polydisk in the $n$-dimensional complex plane $C^{n}$ and let

$$
T^{n}=\left\{z=\left(z_{1}, \ldots, z_{n}\right) \in C^{n},\left|z_{i}\right|=1,1 \leq i \leq n\right\}
$$

be its torus. We denote by $H\left(U^{n}\right)$ the set of holomorphic functions in $U^{n}$ and by $H^{\infty}\left(U^{n}\right)$ the set of bounded holomorphic functions in $U^{n}$.

Let $S$ be the class of all non-negative measurable functions $\omega$ on $(0,1)$, for which there exist positive numbers $M_{\omega}, q_{\omega}, m_{\omega},\left(m_{\omega}, q_{\omega} \in(0,1)\right)$, such that

$$
m_{\omega} \leq \frac{\omega(\lambda r)}{\omega(r)} \leq M_{\omega}
$$

for all $r \in(0,1)$ and $\lambda \in\left[q_{\omega}, 1\right]$. For properties of functions from $S$, see [4]. Using the results of [4], one can prove that

$$
\omega(x)=\exp \left\{\eta(x)+\int_{x}^{1} \frac{\varepsilon(u)}{u} d u\right\}, \quad t \in(0,1),
$$

where $\varepsilon(x), \eta(x)$ are measurable bounded functions and if we set

$$
-\alpha_{\omega}=\frac{\log m_{\omega}}{\log q_{\omega}^{-1}} ; \quad \beta_{\omega}=\frac{\log M_{\omega}}{\log q_{\omega}^{-1}} .
$$

then

$$
-\alpha_{\omega} \leq \varepsilon(u) \leq \beta_{\omega} .
$$


Next we assume that $\eta(x)=0, x \in(0,1)$. We put $\omega(1-|z|)=$ $\prod_{j=1}^{n} \omega_{j}\left(1-\left|z_{j}\right|\right)$ and $(1-\bar{z} z)=\prod_{j=1}^{n}\left(1-\bar{z}_{j} z_{j}\right)$.

Definition 2.1. Let $f \in H\left(U^{n}\right), \omega=\left(\omega_{1}, \ldots, \omega_{n}\right), \omega_{j} \in S, 1 \leq j \leq n$, $0<p \leq 1$. A function $f$ belongs to the space $A^{p}(\omega)\left(\right.$ or $\left.H^{p}(\omega)\right)$ if

$$
\|f\|_{A^{p}(\omega)}^{p}=\int_{U^{n}}|f(z)|^{p} \omega(1-|z|) d m_{2 n}(z)<+\infty,
$$

where $d m_{2 n}(z)$ is the $2 n$-dimensional Lebesgue measure on $U^{n}$ (see [2]).

In particular, if $\omega(t)=t^{\alpha}, \alpha=\left(\alpha_{1}, \ldots, \alpha_{n}\right) \alpha_{j}>-1,1 \leq j \leq n$, then we have $A^{p}(\omega)=A^{p}(\alpha)$ (see [5]).

For $A^{p}(\omega)$ we assume that $0<\beta_{\omega_{j}}<1,1 \leq j \leq n$.

Definition 2.2. Let $\beta=\left(\beta_{1}, \ldots, \beta_{n}\right), \beta_{j}>-1,1 \leq j \leq n$. For a holomorphic function $f(z)=\sum_{|k|=0}^{\infty} a_{k} z^{k}, z \in U^{n}$,

(i) we define the fractional differentiation $D^{\beta}$ in the following way:

$$
\begin{aligned}
& D^{\beta} f(z)=\sum_{|k|=0}^{\infty} \prod_{j=1}^{n} \frac{\Gamma\left(\beta_{j}+1+k_{j}\right)}{\Gamma\left(\beta_{j}+1\right) \Gamma\left(k_{j}+1\right)} a_{k} z^{k}, \quad k=\left(k_{1}, \ldots, k_{n}\right), z \in U^{n}, \\
& D^{\beta} f(\bar{z})=\sum_{|k|=0}^{\infty} \prod_{j=1}^{n} \frac{\Gamma\left(\beta_{j}+1+k_{j}\right)}{\Gamma\left(\beta_{j}+1\right) \Gamma\left(k_{j}+1\right)} a_{k} \bar{z}^{k}, \quad k=\left(k_{1}, \ldots, k_{n}\right), z \in U^{n} .
\end{aligned}
$$

(ii) the inverse operator $D^{-\beta}$ we define in the standard sense: $D^{-\beta} D^{\beta} f(z)=f(z)$.

In particular,

$$
D f(z)=\frac{\partial^{n}(f(z) z)}{\partial z_{1} \ldots \partial z_{n}} .
$$

The following properties of $D$ are valid:

Lemma 2.3. (1) If

$$
h(z)=\int_{U^{n}} \frac{\phi(\zeta) d m_{2 n}(\zeta)}{(1-\bar{\zeta} z)^{\alpha}}, \quad z \in U^{n},
$$

then

$$
\left|D^{\beta} h(z)\right| \leq C(\beta, \pi) \int_{U^{n}} \frac{|\phi(\zeta)| d m_{2 n}(\zeta)}{|1-\bar{\zeta} z|^{\beta+\alpha}}
$$

(2) If $D f(z)=D g(z)$, then $f(z)=g(z), z$ in $U^{n}$. 
Definition 2.4. Let $f \in H\left(U^{n}\right), \omega=\left(\omega_{1}, \ldots, \omega_{n}\right), \omega_{j} \in S, 1 \leq j \leq n$. A function $f$ belongs to the Bloch space $B_{\omega}^{n} \equiv B_{\omega}$ if

$$
M_{f}=\sup _{z \in U^{n}}\left\{\frac{\left(1-|z|^{2}\right)}{\omega\left(1-|z|^{2}\right)}|D f(z)|\right\}<+\infty .
$$

It is easy to see that $B_{\omega}$ is a Banach space with respect to the norm

$$
\|f\|_{B_{\omega}}=|f(0)|+M_{f} .
$$

For $B_{\omega}$ we assume that $0<\alpha_{\omega_{j}}<1,1 \leq j \leq n$.

Proposition 2.5. If $n=1, \omega(t)=t^{1-s}$, then we deal the with known Bloch space of one variable (see [3], [6]).

The proof is evident.

Let $L_{\omega}^{\infty}=L_{\omega}^{\infty}\left(U^{n}\right)$ be the class of measurable functions on $U^{n}$, for which

$$
\|f\|_{L_{\omega}^{\infty}}=\sup _{z \in U^{n}}\left\{|f(z)| \omega\left(1-|z|^{2}\right)\right\}<+\infty .
$$

To prove the main results, we need the following lemmas.

Lemma 2.6. Let $n=1, \omega \in S, a+1-\beta_{\omega}>0, b>1$ and $b-a-2>\alpha_{\omega}$. Then

$$
\int_{U} \frac{\left(1-|w|^{2}\right)^{a} \omega\left(1-|w|^{2}\right)}{|1-z \bar{w}|^{b}} d m_{2}(w) \leq \frac{C \omega\left(1-|z|^{2}\right)}{\left(1-|z|^{2}\right)^{b-a-2}}, \quad C=C(\omega, a, b)
$$

Proof. We have

$$
\int_{-\pi}^{\pi} \frac{d \theta}{\left|1-r \rho e^{i \theta}\right|^{b}} \leq \frac{C(b)}{(1-r \rho)^{b-1}}, \quad b>1 .
$$

Then

$$
\begin{aligned}
& \int_{U} \frac{\left(1-|w|^{2}\right)^{a} \omega\left(1-|w|^{2}\right)}{|1-z \bar{w}|^{b}} d m_{2}(w) \\
& \quad \leq C(b) \int_{0}^{1} \frac{\left(1-\rho^{2}\right)^{a} \omega\left(1-\rho^{2}\right) \rho d \rho d \theta}{(1-r \rho)^{b-1}} \\
& \quad \leq 2^{b-1} C(b) \int_{0}^{1} \frac{(1-t)^{a} \omega(1-t) d t}{\left(1-r^{2} t\right)^{b-1}} \\
& \quad=C_{1}(b) \int_{0}^{1} \frac{u^{a} \omega(u) d u}{\left(1-r^{2}+u r^{2}\right)^{b-1}}
\end{aligned}
$$




$$
\begin{aligned}
& =C_{1}(b)\left\{\int_{0}^{1-r^{2}} \frac{u^{a} \omega(u) d u}{\left(1-r^{2}+u r^{2}\right)^{b-1}}+\int_{1-r^{2}}^{1} \frac{u^{a} \omega(u) d u}{\left(1-r^{2}+u r^{2}\right)^{b-1}}\right\} \\
& =C_{1}(\alpha)\left(I_{1}+I_{2}\right) .
\end{aligned}
$$

First we estimate the integral $I_{1}$ :

$$
\begin{aligned}
I_{1} & \leq \int_{0}^{1-r^{2}} \frac{u^{a} \omega(u)}{\left(1-r^{2}\right)^{b-1}} d u \\
& =\frac{1}{\left(1-r^{2}\right)^{b-1}} \int_{0}^{1-r^{2}} u^{a} \omega(u) d u \\
& =\frac{(a+1)^{-1}}{\left(1-r^{2}\right)^{b-1}}\left[\left(1-r^{2}\right)^{a+1} \omega\left(1-r^{2}\right)+\int_{0}^{1-r^{2}} u^{a} \omega(u) \varepsilon(u) d u\right] .
\end{aligned}
$$

It follows that

$$
(a+1) \int_{0}^{1-r^{2}} u^{a} \omega(u) d u=\left(1-r^{2}\right)^{a+1} \omega\left(1-r^{2}\right)+\int_{0}^{1-r^{2}} u^{a} \omega(u) \varepsilon(u) d u,
$$

and

$$
\int_{0}^{1-r^{2}}(a+1-\varepsilon(u)) u^{a} \omega(u) d u=\left(1-r^{2}\right)^{a+1} \omega\left(1-r^{2}\right) .
$$

On the other hand, we have the inequality

$$
a+1-\beta_{\omega} \leq a+1-\varepsilon(u)
$$

which yields

$$
\left(a+1-\beta_{\omega}\right) \int_{0}^{1-r^{2}} u^{a} \omega(u) d u \leq\left(1-r^{2}\right)^{a+1} \omega\left(1-r^{2}\right)
$$

or

$$
I_{1} \leq C_{1}(\omega) \frac{\omega\left(1-r^{2}\right)}{\left(1-r^{2}\right)^{b-a-2}} .
$$

We now estimate $I_{2}$. For $r^{2} \geq 1 / 2$, we have

$$
\begin{aligned}
I_{2} & \leq 2^{b-1} \int_{1-r^{2}}^{1} \frac{\omega(u)}{u^{b-a-1}} \\
& =\frac{2^{b-1}}{a-b+2} \int_{1-r^{2}}^{1} \omega(u) d u^{a-b+2} \\
& =\frac{2^{b-1}}{b-a-2}\left[\frac{\omega\left(1-r^{2}\right)}{\left(1-r^{2}\right)^{b-a-2}}+\omega(1)+\int_{1-r^{2}}^{1} \frac{\omega(u) \varepsilon(u)}{u^{b-a-1}} d u\right] .
\end{aligned}
$$


Then

$$
\int_{1-r^{2}}^{1} \frac{\omega(u)}{u^{b-a-1}} d u=\frac{\omega\left(1-r^{2}\right)}{\left(1-r^{2}\right)^{b-a-2}}-\omega(1)-\int_{1-r^{2}}^{1} \frac{\omega(u) \varepsilon(u)}{u^{b-a-1}} d u,
$$

and it follows that

$$
\begin{aligned}
\int_{1-r^{2}}^{1}\left(1+\frac{\varepsilon(u)}{b-a-2}\right) \frac{\omega(u)}{u^{b-a-1}} d u & =\frac{\omega\left(1-r^{2}\right)}{\left(1-r^{2}\right)^{b-a-2}}-\omega(1) \\
& \leq \frac{\omega\left(1-r^{2}\right)}{\left(1-r^{2}\right)^{b-a-2}}
\end{aligned}
$$

Then the inequality

$$
1+\frac{\varepsilon(u)}{b-a-2} \geq 1-\frac{\alpha_{\omega}}{b-a-2}>0
$$

gives us

$$
\int_{1-r^{2}}^{1} \frac{\omega(u)}{u^{b-a-1}} d u \leq C_{2}(\omega) \frac{\omega\left(1-r^{2}\right)}{\left(1-r^{2}\right)^{b-a-2}} .
$$

Summing up, from (3) and (4) we get the proof of Lemma 2.6.

Lemma 2.7. Let $\alpha_{j}>\alpha_{\omega_{j}}+1,1 \leq j \leq n$. Then $\left(1-|z|^{2}\right)^{\alpha} D f(z) \in$ $L_{\omega^{-1}}^{\infty}$ if and only if $\left(1-|z|^{2}\right)^{\alpha-1} f(z) \in L_{\omega^{-1}}^{\infty}$.

Proof. Let $g(z)=\left(1-|z|^{2}\right)^{\alpha} D f(z) \in L_{\omega^{-1}}^{\infty}$, then $D f \in A^{1}(\beta)$ for sufficiently large $\beta_{j}, 1 \leq j \leq n$.

From the representation of M. M. Djrbashian (see [5]), we have

$$
D f(z)=\frac{\beta+1}{\pi^{n}} \int_{U^{n}} \frac{\left(1-|\zeta|^{2}\right)^{\beta}}{(1-\bar{\zeta} z)^{\beta+2}} D f(\zeta) d m_{2 n}(\zeta) .
$$

Integrating with respect to $z_{1}$, we obtain

$$
\begin{aligned}
\frac{\partial^{n-1}(f(z) z)}{\partial z_{2} \ldots \partial z_{n}}= & \frac{1}{\pi^{n}} \int_{U^{n}} \frac{\left(1-|\zeta|^{2}\right)^{\beta} D f(\zeta) d m_{2 n}(\zeta)}{\left(1-\bar{\zeta}_{1} z_{1}\right)^{\beta_{1}+1} \bar{\zeta}_{1} \prod_{j=2}^{n}\left(1-\bar{\zeta}_{j} z_{j}\right)^{\beta_{j}+2}} \\
& -\frac{C(\beta)}{\pi^{n}} \int_{U^{n}} \frac{\left(1-|\zeta|^{2}\right)^{\beta} D f(\zeta)}{\bar{\zeta}_{1} \prod_{j=2}^{n}\left(1-\bar{\zeta}_{j} z_{j}\right)^{\beta_{j}+2}} d m_{2 n}(\zeta) .
\end{aligned}
$$

It is easy to see (using for example the Taylor expansion) that the second integral is 0 . Integrating the last equality with respect to $z_{2}, \ldots, z_{n}$, we 
obtain

$$
f(z) z=\frac{1}{\pi^{n}} \int_{U^{n}} \frac{\left(1-|\zeta|^{2}\right)^{\beta}}{(1-\bar{\zeta} z)^{\beta+1} \bar{\zeta}} D f(\zeta) d m_{2 n}(\zeta) .
$$

Next, using the last representation, Lemma 2.6 and maximum modulus principle, we get

$$
\begin{aligned}
|f(z)| & \leq\|g\|_{L_{\omega^{-1}}^{\infty}} \int_{U^{n}} \frac{\left(1-|w|^{2}\right)^{\beta-\alpha} \omega\left(1-|w|^{2}\right)}{|1-\bar{w} z|^{\beta+1}} d m_{2 n}(w) \\
& \leq\|g\|_{L_{\omega^{-1}}^{\infty}} \frac{\omega\left(1-|z|^{2}\right)}{\left(1-|z|^{2}\right)^{\alpha-1}}
\end{aligned}
$$

and therefore $\left(1-|z|^{2}\right)^{\alpha-1} f(z) \in L_{\omega^{-1}}^{\infty}$.

Conversely, if

$$
g_{1}(z)=\left(1-|z|^{2}\right)^{\alpha-1} f(z) \in L_{\omega^{-1}}^{\infty},
$$

then $f \in A^{1}(\beta)$ for $\beta_{j}>\beta_{\omega_{j}}+\alpha_{j}-2$. Lemmas 2.3 and 2.6 imply that

$$
\begin{aligned}
|D f(z)| & \leq C(\beta, \pi) \int_{U^{n}} \frac{\left(1-|\zeta|^{2}\right)^{\beta}}{|1-\bar{\zeta} z|^{\beta+3}}|f(\zeta)| d m_{2 n} \\
& \leq\left\|g_{1}\right\|_{L_{\omega^{-1}}^{\infty}} \int_{U^{n}} \frac{\left(1-|\zeta|^{2}\right)^{\beta-\alpha+1} \omega\left(1-|\zeta|^{2}\right)}{|1-\bar{\zeta} z|^{\beta+3}} \\
& \leq\left\|g_{1}\right\|_{L_{\omega^{-1}}^{\infty}} \frac{\omega\left(1-|z|^{2}\right)}{\left(1-|\zeta|^{2}\right)^{\alpha}} .
\end{aligned}
$$

Thus $\left(1-|z|^{2}\right)^{\alpha} D f(z) \in L_{\omega^{-1}}^{\infty}$.

\section{Main properties of $B_{\omega}$ spaces}

For $\omega_{j} \in S$, we say that $\omega$ satisfies the condition $\Omega$ if

$$
t^{\alpha_{\omega_{j}}} \leq \omega_{j}(t) \leq t^{\gamma_{\omega_{j}}}, \quad \alpha_{\omega_{j}}, \gamma_{\omega_{j}}<0, \quad \text { or } \quad \omega_{j}(t)=\text { const }, \quad 1 \leq j \leq n .
$$

Theorem 3.1. Suppose that $\omega$ satisfies the condition $\Omega$. Then $H^{\infty}\left(U^{n}\right)$ is the subset of $B_{\omega}$.

Proof. Let $f \in H^{\infty}\left(U^{n}\right)$. We consider the torus

$$
\widetilde{T}^{n}=\widetilde{T}_{1} \ldots \widetilde{T}_{n}, \quad \widetilde{T}_{j}=\left\{\zeta_{j}, \zeta_{j}=z_{j}+\eta_{j} \frac{\left(1-\left|z_{j}\right|^{2}\right)}{\omega_{j}\left(1-\left|z_{j}\right|^{2}\right)} e^{i \theta_{j}}\right\} \quad j=1, \ldots, n .
$$


We take $\eta_{j}$ so that $\widetilde{T}_{j}$ lies in the unit disc. Using the Cauchy formula for $\widetilde{T}^{n}$, we get

$$
D f(z)=\frac{\partial^{n}(f(z) z)}{\partial z_{1} \ldots \partial z_{n}}=\frac{1}{(2 \pi i)^{n}} \int_{\widetilde{T}^{n}} \frac{f(\zeta) \zeta d \zeta}{(\zeta-z)^{2}} .
$$

It follows that

$$
\begin{aligned}
\left|\frac{\partial^{n}(f(z) z)}{\partial z_{1} \ldots \partial z_{n}}\right| & \leq \frac{1}{(2 \pi)^{n}} \int_{\widetilde{T}^{n}} \frac{|f(\zeta) \zeta| \prod_{j=1}^{n} \eta_{j} \frac{\left(1-\left|z_{j}\right|^{2}\right)}{\omega_{j}\left(1-\left|z_{j}\right|^{2}\right)} d \theta_{1} \ldots d \theta_{n}}{\prod_{j=1}^{n} \eta_{j}^{2} \frac{\left(1-\left|z_{j}\right|^{2}\right)^{2}}{\omega_{j}^{2}\left(1-\left|z_{j}\right|^{2}\right)}} \\
& \leq C \int_{\widetilde{T}^{n}} \frac{d \theta_{1} \ldots d \theta_{n}}{\prod_{j=1}^{n} \eta_{j} \frac{1-\left|z_{j}\right|^{2}}{\omega_{j}\left(1-\left|z_{j}\right|^{2}\right)}} \\
& =C_{2} \frac{\omega\left(1-|z|^{2}\right)}{1-|z|^{2}}
\end{aligned}
$$

and hence $f \in B_{\omega}\left(U^{n}\right)$.

Remark 3.2. Note that $B_{\omega} \not \subset H^{\infty}$. As an example we can take the function $f(z)=\log \left(1-z_{1}\right) \ldots \log \left(1-z_{n}\right)$.

Theorem 3.3. Let $\omega$ satisfy the condition $\Omega$. Then the classes $B_{\omega}$ are invariant with respect to multiplication by monomials: $f \in B_{\omega}$ if and only if $z_{j} f \in B_{\omega}$ for any $j, j \in[1, n]$.

Proof. Let $f \in B_{\omega}$ and $j=1$. Then

$$
\frac{\partial^{n}\left(f(z) z z_{1}\right)}{\partial z_{1} \ldots \partial z_{n}}=\frac{\partial^{n}(f(z) z)}{\partial z_{1} \ldots \partial z_{n}} z_{1}+\frac{\partial^{n-1}(f(z) z)}{\partial z_{2} \ldots \partial z_{n}} .
$$

Using the fact that $h(z) z=\int_{0}^{z}(h(t) t)^{\prime} d t, h \in H(U)$, we get

$$
\left|\frac{\partial^{n-1}(f(z) z)}{\partial z_{2} \ldots \partial z_{n}}\right| \leq M_{f} \frac{\omega\left(1-|z|^{2}\right)}{\left(1-|z|^{2}\right)} .
$$

It follows that $f(z) z_{1} \in B_{\omega}$.

Conversely, let $f(z) z_{1} \in B_{\omega}$. We will prove that $f \in B_{\omega}$. We have $f(z)=\frac{1}{z_{1}} f(z) z_{1}$ and

$$
D f(z)=\frac{\partial^{n}(f(z) z)}{\partial z_{1} \ldots \partial z_{n}}=-\frac{1}{z_{1}^{2}} \frac{\partial^{n-1}\left(f(z) z z_{1}\right)}{\partial z_{2} \ldots \partial z_{n}}+\frac{1}{z_{1}} \frac{\partial^{n}\left(f(z) z z_{1}\right)}{\partial z_{1} \ldots \partial z_{n}} .
$$


Therefore,

$$
|D f(z)| \leq \frac{1}{\left|z_{1}\right|^{2}}\left|\frac{\partial^{n-1}\left(f(z) z z_{1}\right)}{\partial z_{2} \ldots \partial z_{n}}\right|+\frac{1}{\left|z_{1}\right|}\left|\frac{\partial^{n}\left(f(z) z z_{1}\right)}{\partial z_{1} \ldots \partial z_{n}}\right| .
$$

If $\left|z_{1}\right| \geq \frac{1}{2}$, then

$$
|D f(z)| \leq 4\left|\frac{\partial^{n-1}\left(f(z) z z_{1}\right)}{\partial z_{2} \ldots \partial z_{n}}\right|+2\left|\frac{\partial^{n}\left(f(z) z z_{1}\right)}{\partial z_{1} \ldots \partial z_{n}}\right|
$$

and

$$
\frac{\left(1-|z|^{2}\right)}{\omega\left(1-|z|^{2}\right)}|D f(z)|<6 M_{f}<\infty
$$

If $\left|z_{1}\right|<1 / 2$, then we use maximum of modulus principle for the holomorphic function $\frac{\partial^{n} f(z)}{\partial z_{1} \ldots \partial z_{n}} z_{1}^{2}$. Thus, (6) is true for all $\left|z_{1}\right|<1$ and $f \in B_{\omega}$.

Proposition 3.4. If $\omega$ satisfies the condition $\Omega$, then $B_{\omega}$ is an algebra.

Proof. The proof is standard. It is evident that if $f, g \in B_{\omega}$, then $\alpha f+\beta f \in B_{\omega}$ for all $\alpha, \beta \in C$. Let us show that $f g \in B_{\omega}$. To this end, we use the Leibniz formula

$$
\frac{\partial^{k}(f(z) g(z))}{\partial z_{1} \ldots \partial z_{k}}=\sum_{j=0}^{k} C_{k}^{j} \frac{\partial^{j} f(z)}{\partial z_{i_{1}} \ldots \partial z_{i_{j}}} \cdot \frac{\partial^{k-j} g(z)}{\partial z_{i_{j+1}} \ldots \partial z_{i_{k}}} .
$$

and the fact that $f, g \in B_{\omega}$. It follows that $f \cdot g \in B_{\omega}$.

The following proposition shows that, in some cases, the spaces $B_{\omega}$ are $\omega$ weighted Lipschitz classes (see [7]).

Proposition 3.5. Let $t^{\alpha_{\omega_{j}}} \leq \omega_{j}(t) \leq t^{\gamma_{\omega_{j}}}, 0<\alpha_{\omega_{j}}, \gamma_{\omega_{j}}<1,1 \leq j \leq n$ then $B_{\omega}=\widetilde{\Lambda}_{a}(\omega)$, where $\widetilde{\Lambda}_{a}(\omega)$ denotes the weighted Lipschitz spaces for polydisk.

Proof. Using (1), it is not difficult to see that there exist $C_{1}, C_{2} \neq 0$ such that

$$
C_{1} \omega_{j}\left(1-\left|z_{j}\right|\right) \leq \omega_{j}\left(1-\left|z_{j}\right|^{2}\right) \leq C_{1} \omega_{j}\left(1-\left|z_{j}\right|\right), \quad j=1,2, \ldots, n .
$$

Let $f \in B_{\omega}$ and $\left(i_{i}, \ldots, i_{k}\right)=(1, \ldots, k)$. Then

$$
|D f(z)| \leq M_{f} \frac{\omega(1-|z|)}{(1-|z|)} .
$$


Integrating this inequality with respect to $z_{k+1}, \ldots, z_{n}$, we derive

$$
\left|\frac{\partial^{k}\left(f(z) z^{\prime}\right)}{\partial z_{1} \ldots \partial z_{k}}\right| \leq C_{2} M_{f} \prod_{j=1}^{k} \frac{\omega_{j}\left(1-\left|z_{j}\right|\right)}{\left(1-\left|z_{j}\right|\right)} \quad z^{\prime}=\left(z_{1}, . ., z_{k}\right) .
$$

From the invariance of $\widetilde{\Lambda}_{a}(\omega)$ with respect to the monomial multiplication, it follows that $f \in \widetilde{\Lambda}_{a}(\omega)$.

Let now $f \in \widetilde{\Lambda}_{a}(\omega)$. Using the characterisation and invariance with respect to the monomial multiplication of holomorphic Lipschitz classes again, we see that $f \in B_{\omega}$.

Hence, in view of the results of [7], we obtain

Corollary 3.6. Let $t^{\alpha_{\omega_{j}}} \leq \omega_{j}(t) \leq t^{\gamma_{\omega_{j}}}, 0<\alpha_{\omega_{j}}, \gamma_{\omega_{j}}<1,1 \leq j \leq n$. Then:

(i) $B_{\omega}$, is an algebra and $B_{\omega} \subset H^{\infty}\left(U^{n}\right)$;

(ii) $B_{\omega}$ is invariant with respect monomial multiplication.

\section{Projection theorems and diagonal mappings}

For $\alpha=\left(\alpha_{1}, \ldots, \alpha_{n}\right), \alpha_{j}>0,1 \leq j \leq n$, we introduce the operator

$$
Q_{\alpha} f(z)=\alpha \int_{U^{n}} \frac{f(\zeta)}{(1-\bar{\zeta} z)^{\alpha+1}} d m_{2 n}(\zeta)
$$

which is not a projection.

Theorem 4.1. Let $\alpha_{j}>\beta_{\omega_{j}}, 1 \leq j \leq n$. Then the map $Q_{\alpha}$ is bounded from $L_{\widetilde{\omega}^{-}}^{\infty}$ to $B_{\omega}$, where $\widetilde{\omega}(t)=t^{\alpha-1} \omega(t)$. Moreover, if $\alpha_{j}>$ $\max \left\{\beta_{\omega_{j}}+1, \alpha_{\omega_{j}}+1\right\}, 1 \leq j \leq n$ then $Q_{\alpha}$ is surjective.

Proof. We show that (2) is true for the function $F(z)=Q_{\alpha} f(z)$ :

$$
\begin{aligned}
|D F(z)| & \leq \alpha \int_{U^{n}} \frac{|f(w)| \omega\left(1-|w|^{2}\right)}{|1-\bar{w} z|^{\alpha+2} \omega\left(1-|w|^{2}\right)} d m_{2 n}(w) \\
& \leq \alpha\|f\|_{L_{\tilde{\omega}^{-1}}^{\infty}} \int_{U^{n}} \frac{\left(1-|w|^{2}\right)^{\alpha-1} \omega\left(1-|w|^{2}\right)}{|1-\bar{w} z|^{\alpha+2}} d m_{2 n}(w) .
\end{aligned}
$$

Using Lemma 2.6, we get

$$
\|F\|_{B_{\omega}} \leq C(\omega, \alpha)\|f\|_{L_{\tilde{\omega}-1}^{\infty}}
$$

and we see that $Q_{\alpha}$ is a bounded operator from $L_{\widetilde{\omega}^{-1}}^{\infty}$ into $B_{\omega}$. 
Next we show that $Q_{\alpha}$ is surjective: for any $f \in B_{\omega}$ there exists a function $h \in L_{\widetilde{\omega}^{-1}}^{\infty}$ so that $f(z)=Q_{\alpha} h(z)$.

Let $f \in B_{\omega}$ and $\alpha_{j}>\max \left\{\beta_{\omega_{j}}+1, \alpha_{\omega_{j}}+1\right\}, 1 \leq j \leq n$. The function $\frac{\alpha+1}{\alpha \pi^{n}}\left(1-|z|^{2}\right)^{\alpha} D f(z)$ belongs to $L_{\widetilde{\omega}^{-1}}^{\infty}$. Using Lemma 2.7 , we see that the function $h(z)=\frac{\alpha-1}{\alpha \pi^{n}}\left(1-|z|^{2}\right)^{\alpha-1} f(z)$ also belongs to $L_{\widetilde{\omega}^{-1}}^{\infty}$. We set

$$
Q_{\alpha} h_{1}(z)=\frac{\alpha-1}{\pi^{n}} \int_{U^{n}} \frac{\left(1-|w|^{2}\right)^{\alpha-1}}{(1-\bar{w} z)^{\alpha+1}} f(w) d m_{2 n}(w) .
$$

On the other hand, the function $f(z)$ is holomorphic and belongs to $A^{1}(\alpha-1)$ if $\alpha_{j}>\beta_{\omega_{j}}+1,1 \leq j \leq n$. It can be written as

$$
f(z)=\frac{\alpha-1}{\pi^{n}} \int_{U^{n}} \frac{\left(1-|w|^{2}\right)^{\alpha-1}}{(1-\bar{w} z)^{\alpha+1}} f(w) d m_{2 n}(w) .
$$

Thus, we get $f(z)=Q_{\alpha} h(z) \quad z \in U^{n}$.

Consider now the projection

$$
P_{\alpha} f(z)=\frac{(\alpha+1)}{\pi^{n}} \int_{U^{n}} \frac{\left(1-|w|^{2}\right)^{\alpha}}{(1-\bar{w} z)^{\alpha+2}} f(w) d m_{2 n}(w)\left(\alpha_{j}>0,1 \leq j \leq n\right),
$$

it is not diffucult to prove the following result:

Proposition 4.2. Let $\alpha_{j}>\beta_{\omega_{j}}, 1 \leq j \leq n$. Then $P_{\alpha}: B_{\omega} \rightarrow L_{\omega^{-1}}^{\infty}$ and is a bounded.

The question is whether there is an inverse operator $R_{\alpha, \beta}$ which maps $B_{\omega}$ to $L_{\omega^{-1}}^{\infty}$ and furthermore, if this is the case, whether $P_{\alpha}\left(R_{\alpha, \beta}(f)\right)(z)=f(z)$ for all $f \in B_{\omega}$. The answer is positive.

Let us define the 'inverse' operator as

$$
\begin{gathered}
R_{\alpha, \beta} f(z)=\left(1-|z|^{2}\right)^{\beta} \int_{U^{n}} \frac{\left(1-|\zeta|^{2}\right)^{\alpha-1}}{(1-\bar{\zeta} z)^{\alpha+\beta+1}} f(\zeta) d m_{2 n}(\zeta), \\
\alpha=\left(\alpha_{1}, \ldots, \alpha_{n}\right) \beta=\left(\beta_{1}, \ldots, \beta_{n}\right), \alpha_{j}>0, \beta_{j} \geq 1,1 \leq j \leq n .
\end{gathered}
$$

We first show that $P_{\alpha} R_{\alpha, \beta} f(z)=f(z), z \in U^{n}$. To this end, let us calculate $P_{\alpha} R_{\alpha, \beta} f(z)$ using the Fubini theorem:

$$
\begin{aligned}
P_{\alpha} R_{\alpha, \beta} f(z)= & \frac{\alpha}{\pi^{n}} \int_{U^{n}} \frac{\left(1-|\zeta|^{2}\right)^{\alpha+\beta-1}}{(1-\bar{\zeta} z)^{\alpha+1}} \\
& \times \int_{U^{n}} \frac{\left(1-|w|^{2}\right)^{\alpha-1} f(w)}{(1-\bar{w} \zeta)^{\alpha+\beta+1}} d m_{2 n}(w) d m_{2 n}(\zeta) \\
= & \frac{\alpha+1}{\pi^{n}} \int_{U^{n}}\left(1-|w|^{2}\right)^{\alpha-1} f(w)
\end{aligned}
$$




$$
\begin{aligned}
& \times \int_{U^{n}} \frac{\left(1-|\zeta|^{2}\right)^{\alpha+\beta-1} d m_{2 n}(\zeta)}{(1-\bar{\zeta} z)^{\alpha+1}(1-\bar{w} \zeta)^{\alpha+\beta+1}} d m_{2 n}(w) \\
= & \int_{U^{n}} \frac{\left(1-|w|^{2}\right)^{\alpha-1}}{(1-\bar{w} z)^{\alpha+1}} f(w) d m_{2 n}(w)=f(z), \quad\left(\alpha_{j}>\beta_{\omega_{j}}+1\right) .
\end{aligned}
$$

Theorem 4.3. Let $\alpha_{j}>\max \left\{\beta_{\omega_{j}}+1, \alpha_{\omega_{j}}+1\right\}$ and $\beta_{j}>\alpha_{\omega_{j}}, 1 \leq j \leq n$. Then

(a) The operator $R_{\alpha, \beta}$ is bounded from $B_{\omega}$ to $L_{\omega^{-1}}^{\infty}$, and there exist constants $C_{1}(\omega), C_{1}(\omega)$ such that

$$
C_{1}(\omega)\|f\|_{B_{\omega}} \leq\left\|R_{\alpha, \beta} f\right\|_{L_{\omega^{-1}}^{\infty}} \leq C_{2}(\omega)\|f\|_{B_{\omega}} .
$$

(b) $f \in B_{\omega}$ if and only if $R_{\alpha, \beta} f \in L_{\omega^{-1}}^{\infty}$.

Proof. (a) Let $f \in B_{\omega}$. Theorem 4.1 implies that there exists a function $g \in L_{\widetilde{\omega}^{-1}}^{\infty}$ such that $Q_{\alpha} g(z)=f(z), \quad z \in U^{n}$. Using the Fubini theorem, we get

$$
\begin{aligned}
R_{\alpha, \beta} f(z) & =\alpha\left(1-|z|^{2}\right)^{\beta} \int_{U^{n}} g(w) \int_{U^{n}} \frac{\left(1-|\zeta|^{2}\right)^{\alpha-1} d m_{2 n}(\zeta)}{(1-\bar{\zeta} z)^{\alpha+\beta+1}(1-\bar{w} \zeta)^{\alpha+1}} d m_{2 n}(w) \\
& =\pi^{n}\left(1-|z|^{2}\right)^{\beta} \int_{U^{n}} \frac{g(w)}{(1-\bar{w} z)^{\alpha+\beta+1}} d m_{2 n}(w) .
\end{aligned}
$$

Thus,

$$
\begin{aligned}
\left|R_{\alpha, \beta} f(z)\right| & \leq\|g\|_{L_{\tilde{\omega}^{-1}}^{\infty}}\left(1-|z|^{2}\right)^{\beta} \int_{U^{n}} \frac{\left(1-|w|^{2}\right)^{\alpha-1} \omega\left(1-|w|^{2}\right)}{|1-\bar{w} z|^{\alpha+\beta+1}} d m_{2 n}(w) \\
& \leq C(\omega)\|g\|_{L_{\tilde{\omega}^{-1}}^{\infty}} \omega\left(1-|z|^{2}\right),
\end{aligned}
$$

and hence

$$
\left\|R_{\alpha, \beta} f\right\|_{L_{\omega^{-1}}^{\infty}} \leq C(\omega)\|g\|_{L_{\tilde{\omega}^{-1}}^{\infty}}, g \in L_{\widetilde{\omega}^{-1}}^{\infty} .
$$

Using Theorem 4.1 and the open mapping theorem, we get

$$
\left\|R_{\alpha, \beta} f\right\|_{L_{\omega^{-1}}^{\infty}} \leq C_{2}(\omega)\|f\|_{B_{\omega}}
$$

i.e. $\quad R_{\alpha, \beta}$ is bounded from $B_{\omega}$ to $L_{\omega^{1}-}^{\infty}$. To prove the other inequality in (7), we use the fact, that $P_{\alpha} R_{\alpha, \beta} f(z)=f(z)$ for all $f \in B_{\omega}$. Then by Proposition 4.2, there exists $C_{0}>0$ such that

$$
\|f\|_{B_{\omega}}=\left\|P_{\alpha} R_{\alpha, \beta} f\right\|_{B_{\omega}} \leq C_{0}(\omega)\left\|R_{\alpha, \beta} f\right\|_{L_{\omega}^{\infty}-1}
$$

Taking $C_{1}=C_{0}^{-1}$, we obtain the left-hand side inequality in (7).

(b) The proof follows from (8) and (9). 
Let us formulate the problem of diagonal mapping in the general case: let $f \in X \subset H\left(U^{n}\right)$, then the function $g(z)=f^{*}(z)=f(z, \ldots, z)$ is analytic on the unit disk.

Problem. Find all functions $g$ of the class $H\left(U^{n}\right)$ for which there is $f \in X$, such that

$$
g(z)=f^{*}(z)=f(z, \ldots, z) .
$$

Assume that $n=2, X=B_{\omega}$. Then we have the following theorem

Theorem 4.4. Let $\omega_{1}, \omega_{2} \in S$ and $f \in B^{2}(\omega)$. Then $f^{*} \in B_{\omega^{*}}^{1}$, where $\omega^{*}(t)=\omega_{1}(t) \omega_{2}(t)$. Conversely, for any $g \in B_{\omega^{*}}^{1}$ there exists a function $f \in B_{\omega}$ such that $g(z)=f^{*}(z), \quad z \in U$.

Proof. Let $f \in B^{2}(\omega)$, then

$$
M_{f}=\sup _{z \in U}\left\{\frac{\left(1-|z|^{2}\right)^{2}}{\omega_{1}\left(1-|z|^{2}\right) \omega_{2}\left(1-|z|^{2}\right)}\left|D^{2} g(z)\right|\right\}<+\infty
$$

and hence $g \in B_{\omega^{*}}^{1}$.

Now show that for any $g \in B_{\omega^{*}}^{1}$ there exists $f \in B_{\omega}$ so that $g(z)=f^{*}(z)$. Indeed, taking $\gamma>\left(\beta_{\omega_{1}}+\beta_{\omega_{2}}+2\right) / 2$ it is easy to show that $D g(z) \in$ $A^{1}(\gamma n-2)$ and, hence,

$$
D g(z)=C(\gamma, \pi) \int_{U} \frac{\left(1-|\zeta|^{2}\right)^{2 \gamma-2}}{(1-\bar{\zeta} z)^{2 \gamma}} D g(\zeta) d m_{2}(\zeta) .
$$

Let us write

$$
D f(z)=C(\gamma, \pi) \int_{U} \frac{\left(1-|\zeta|^{2}\right)^{2 \gamma-2}}{\left(1-\bar{\zeta} z_{1}\right)^{\gamma}\left(1-\bar{\zeta} z_{2}\right)^{\gamma}} D g(\zeta) d m_{2}(\zeta) .
$$

Then $f(z)=D^{-}(D f(z))$ and $D f^{*}(z)=D g(z)$. Therefore from Lemma 2.3 we get $f^{*}(z)=g(z), \quad z \in U$.

Let us show that $f \in B_{\omega}$. In fact,

$$
\begin{aligned}
|D f(z)| \leq C(\gamma, \pi) & \leq \int_{U} \frac{\left(1-|\zeta|^{2}\right)^{2 \gamma-2}|D g(z)|}{\left|1-\bar{\zeta} z_{1}\right| \gamma\left|1-\bar{\zeta} z_{2}\right|^{\gamma}} d m_{2}(\zeta) \\
& \leq\|g\|_{B_{\omega^{*}}} \int_{U} \frac{\left(1-|\zeta|^{2}\right)^{2 \gamma-3} \mid \omega^{*}\left(1-|\zeta|^{2}\right)}{\left|1-\bar{\zeta} z_{1}\right|^{\gamma}\left|1-\bar{\zeta} z_{2}\right|^{\gamma}} d m_{2}(\zeta) .
\end{aligned}
$$

Using Holder inequality and Lemma 2.6, we get

$$
|D f(z)| \leq C^{*}\left(\int_{U} \frac{\left(1-|\zeta|^{2}\right)^{2 \gamma-3} \mid \omega_{1}^{2}\left(1-|\zeta|^{2}\right)}{\left|1-\bar{\zeta} z_{1}\right|^{2 \gamma}} d m_{2}(\zeta)\right)^{1 / 2}
$$




$$
\begin{aligned}
& \times\left(\int_{U} \frac{\left(1-|\zeta|^{2}\right)^{2 \gamma-3} \mid \omega_{2}^{2}\left(1-|\zeta|^{2}\right)}{\left|1-\bar{\zeta} z_{2}\right|^{2 \gamma}} d m_{2}(\zeta)\right)^{1 / 2} \\
\leq & \frac{\omega_{1}\left(1-\left|z_{1}\right|^{2}\right) \omega_{2}\left(1-\left|z_{2}\right|^{2}\right)}{\left(1-\left|z_{1}\right|\right)\left(1-\left|z_{2}\right|\right)}
\end{aligned}
$$

Hence $f \in B_{\omega}^{2}$.

\section{Linear continuous functionals on $A^{p}(\omega)$}

We need to establish the following theorem before proving the duality result.

Theorem 5.1. Let $\beta_{j}>0,1 \leq j \leq n$. Then $f \in B_{\omega}$ if and only if

$$
M_{f}^{\beta}=\sup _{z \in U^{n}}\left\{\frac{\left(1-|z|^{2}\right)^{\beta+1}}{\omega\left(1-|z|^{2}\right)} D^{\beta+1} f(z) \mid\right\}<\infty .
$$

Proof. Let $f \in B_{\omega}$. Then, by Theorem 4.1, there exists a function $g \in L_{\widetilde{\omega}^{-1}}^{\infty}$ such that

$$
f(z)=\alpha \int_{U^{n}} \frac{g(w)}{(1-\bar{w} z)^{\alpha+1}} d m_{2 n}(w) .
$$

For $\beta_{j}+1>\alpha_{\omega_{j}}$, we get

$$
\begin{aligned}
\left|D^{\beta+1} f(z)\right| & \leq \alpha \int_{U^{n}} \frac{|g(w)| \omega\left(1-|w|^{2}\right)}{|1-\bar{w} z|^{\alpha+\beta+2} \omega\left(1-|w|^{2}\right)} d m_{2 n}(w) \\
& \leq \alpha\|g\|_{L_{\tilde{\omega}^{-1}}^{\infty}} \int_{U^{n}} \frac{\left(1-|w|^{2}\right)^{\alpha} \omega\left(1-|w|^{2}\right)}{|1-\bar{w} z|^{\alpha+\beta+3}} d m_{2 n}(w) \\
& \leq C(\alpha, \beta, \omega)\|g\|_{L_{\tilde{\omega}^{-1}}^{\infty}} \frac{\omega\left(1-|z|^{2}\right)}{\left(1-|z|^{2}\right)^{\beta+1}}
\end{aligned}
$$

It follows that

$$
\sup _{z \in U^{n}}\left\{\frac{\left(1-|z|^{2}\right)^{\beta+1}}{\omega\left(1-|z|^{2}\right)} D^{\beta+1} f(z) \mid\right\}<\infty .
$$

Conversely, if (10) takes place, then taking $m_{j}, 1 \leq j \leq n$ sufficiently large, using the inequality

$$
|D f(z)| \leq C(m, \pi, \beta) \int_{U^{n}} \frac{\left(1-|\zeta|^{2}\right)^{m}}{|1-\bar{\zeta} z|^{m+2-\beta}}\left|D^{\beta+1} f(\zeta)\right| d m_{2 n}(\zeta),
$$


and applying Lemma 2.6, we easily show that

$$
\sup _{z \in U^{n}}\left\{\frac{\left(1-|z|^{2}\right)}{\omega\left(1-|z|^{2}\right)} D f(z) \mid\right\}<\infty .
$$

The following theorem describes the continuous linear functionals on $A^{p}(\omega)$.

Theorem 5.2. Let $\Phi$ be a continuous linear functional on $A^{p}(\omega), 0<$ $p \leq 1$ and let $g(z)=\Phi\left((1-z w)^{-1}\right),\left(z, w \in U^{n}\right)$. Then:

(i) (a) $g \in B_{\widetilde{\omega}}, \widetilde{\omega}(t)=\omega^{1 / p}(t) t^{2 / p-1}$

(b) The functional $\Phi$ is representable in the forms

$$
\begin{gathered}
\Phi(f)=\lim _{\rho \rightarrow 1-0} \frac{1}{(2 \pi)^{n}} \int_{\mathbb{T}^{n}} f(\rho \zeta) g(\rho \bar{\zeta}) d m_{n}(\zeta), \\
\Phi(f)=\lim _{\rho \rightarrow 1-0} \frac{1}{(2 \pi)^{n}} \int_{U^{n}} f(\zeta) D^{m+1} g\left(\rho^{2} \bar{\zeta}\right)\left(1-|\zeta|^{2}\right)^{m} d m_{2 n}(\zeta), \\
m=\left(m_{1}, \ldots, m_{n}\right), m_{j}>\frac{\alpha_{\omega_{j}}+2}{p}, 1 \leq j \leq n .
\end{gathered}
$$

Moreover, there are positive constants $C_{1}(\widetilde{\omega})$ and $C_{2}(\omega)$ such that

$$
C_{1}(\widetilde{\omega})\|\Phi\| \leq\|g\|_{B_{\tilde{\omega}}} \leq C_{2}(\widetilde{\omega})\|\Phi\| .
$$

(ii) Conversely, for any function $g \in B_{\widetilde{\omega}}$ relations (11) and (12) define a continuous linear functional on $A^{p}(\omega)$, which satisfies (13).

Proof. It is easy to show that the linear functional $\Phi$ is continuous on $A^{p}(\omega)$ if and only if

$$
\|\Phi\|=\sup _{\|f\|_{A^{p}(\omega)} \leq 1}|\Phi(f)|<+\infty .
$$

(i) Let $\Phi$ be a continuous linear functional on the space $A^{p}(\omega)$ and let $e_{z}=(1-\zeta z)^{-1}\left(\right.$ remember $\left.0<\beta_{\omega_{j}}<1,0 \leq j \leq n\right)$. Then

$$
\begin{aligned}
\left|D^{\beta+1} g(z)\right| & =\left|\Phi\left(D^{\beta+1} e_{z}\right)\right| \leq\|\Phi\|\left\|D^{\beta+1} e_{z}\right\|_{A^{p}(\omega)} \\
& =\|\Phi\|\left(\int_{U^{n}} \frac{\omega\left(1-|\zeta|^{2}\right)}{|1-\zeta z|^{p(\beta+2)}} d m_{2 n}(\zeta)\right)^{1 / p} \\
& \leq C_{2}(\widetilde{\omega})\|\Phi\| \frac{\omega^{1 / p}\left(1-|z|^{2}\right)}{\left(1-|z|^{2}\right)^{\beta+2-2 / p}} .
\end{aligned}
$$


We get

$$
\left|D^{\beta+1} g(z)\right| \frac{\left(1-|z|^{2}\right)^{\beta+2-2 / p}}{\omega^{1 / p}\left(1-|z|^{2}\right)} \leq C_{2}(\widetilde{\omega})\|\Phi\| .
$$

Hence, by Theorem 5.1, we have $g \in B_{\widetilde{\omega}}$ and $\|g\|_{B_{\widetilde{\omega}}} \leq C_{2}(\widetilde{\omega})\|\Phi\|$.

We now show that $\Phi$ has the form (11). We have

$$
g(z)=\Phi\left(e_{z}\right)=\sum_{|k|=0}^{\infty} \Phi\left(\zeta^{k}\right) z^{k}=\sum_{|k|=0}^{\infty} a_{k} z^{k}, \quad a_{k}=\Phi\left(\zeta^{k}\right) .
$$

It is well known, that

$$
\lim _{\rho \rightarrow 1-0}\left\|f-f_{\rho}\right\|_{A^{p}(\omega)} \rightarrow 0, \quad f \in A^{p}(\omega), \quad f_{\rho}(z)=f(\rho z) .
$$

Thus

$$
\Phi(f)=\lim _{\rho \rightarrow 1-0} \Phi\left(f_{\rho}\right)=\lim _{\rho \rightarrow 1-0} \sum_{|k|=0}^{\infty} a_{k} b_{k} \rho^{k}, \quad\left(f(z)=\sum_{|k|=0}^{\infty} b_{k} z^{k}\right) .
$$

We can also write

$$
\Phi(f)=\lim _{\rho \rightarrow 1-0} \sum_{|k|=0}^{\infty} a_{k} b_{k} \rho^{2 k} .
$$

Calculations show that

$$
\int_{T^{n}} f(\rho \zeta) g(\rho \bar{\zeta}) d m_{n}(\zeta)=\sum_{|k|=0}^{\infty} a_{k} b_{k} \rho^{2 k}
$$

which proves (11).

(ii) Let us show that for any function $g \in B_{\widetilde{\omega}}$ relations (11)and (12) define a continuous linear functional on $A^{p}(\omega)$, which satisfies (13). Let $f \in A^{p}(\omega)$. Then $f \in A^{1}(m)$ for some sufficiely large $m=\left(m_{1}, \ldots, m_{n}\right)$ and from the Djrbashian representation we get

$$
\begin{aligned}
\int_{T^{n}} f(\rho \zeta) g(\rho \bar{\zeta}) d m_{n}(\zeta)= & \frac{m+1}{\pi^{n}} \int_{T^{n}} g(\rho \bar{\zeta}) \\
& \times \int_{U^{n}} \frac{\left(1-|w|^{2}\right)^{m} f(w)}{(1-\rho \bar{w} \zeta)^{m+2}} d m_{2 n}(w) d m_{n}(\zeta) \\
= & \frac{m+1}{\pi^{n}} \int_{U^{n}} f(w)\left(1-|w|^{2}\right)^{m} \\
& \times \int_{T^{n}} \frac{g(\rho \bar{\zeta})}{\left(1-\bar{w} \rho \zeta^{m+2}\right.} d m_{n}(\zeta) d m_{2 n}(w) .
\end{aligned}
$$


Nothing that

$$
\frac{1}{(2 \pi)^{n}} \int_{T^{n}} \frac{g(\rho \bar{\zeta})}{(1-\bar{w} \rho \zeta)^{m+2}} d m_{n}(\zeta)=D^{m+1} g\left(\rho^{2} \bar{w}\right)
$$

we obtain the formula

$$
\int_{T^{n}} f(\rho \zeta) g(\rho \bar{\zeta}) d m_{n}(\zeta)=\int_{U^{n}} f(\zeta) D^{m+1} g\left(\rho^{2} \bar{\zeta}\right)\left(1-|\zeta|^{2}\right)^{m} d m_{2 n}(\zeta)
$$

To show that the limits in (11) exist, we estimate the right hand-side of (14):

$$
I=\int_{U^{n}} f(\zeta) D^{m+1} g\left(\rho^{2} \bar{\zeta}\right)\left(1-|\zeta|^{2}\right)^{m} d m_{2 n}(\zeta)
$$

We have

$$
I \leq C\|g\|_{B_{\tilde{\omega}}} \int_{U^{n}} \frac{\omega^{1 / p}\left(1-|\zeta|^{2}\right)}{\left(1-|\zeta|^{2}\right)^{2-2 / p}}|f(\zeta)| d m_{2 n}(\zeta) .
$$

Let $k=\left(k_{1}, . ., k_{n}\right), k_{j} \geq 0, l_{j} \in Z$ and $-2^{k_{j}} \leq l_{j} \leq 2^{k_{j}+1}-1,1 \leq j \leq n$. We put

$$
\begin{gathered}
\Delta_{k_{j}, l_{j}}=\left\{z_{j}: 1-1 / 2^{k_{j}} \leq\left|z_{j}\right|<1-1 / 2^{k_{j}+1}, \pi l_{j} / 2^{k_{j}} \leq \arg z_{j}<\pi\left(l_{j}+1\right) / 2^{k_{j}}\right\} \\
\Delta_{k, l}=\Delta_{k_{1}, l_{1}} \times \ldots \times \Delta_{k_{n}, l_{n}}
\end{gathered}
$$

Since $0<p \leq 1$, we have

$$
\begin{gathered}
I^{p} \leq C_{1}\|g\|_{B_{\widetilde{\omega}}}^{p} \sum_{|k|=0}^{\infty} \sum_{j=-2^{k}}^{2^{k}-1} \max _{\zeta \in \bar{\Delta}_{k, j}}|f(\zeta)|^{p} \frac{\omega\left(1-\left|\zeta_{k, j}\right|\right)}{\left(1-\left|\zeta_{k, j}\right|\right)^{p-2}}\left|\Delta_{k, j}\right|^{p} \\
k=\left(k_{1}, \ldots, k_{n}\right), \quad j=\left(j_{1}, \ldots, j_{n}\right), \quad 2^{k}=\left(2^{k_{1}}, \ldots, 2^{k_{n}}\right) .
\end{gathered}
$$

Hence

$$
\begin{gathered}
(1-\eta)\left(1-\left|\zeta_{k_{l}, j_{l}}\right|\right) \leq 1-\left|\zeta_{l}\right| \leq(1+\eta)\left(1-\left|\zeta_{k_{l}, j_{l}}\right|\right), \quad \zeta_{l} \in \Delta_{k, j} \\
\frac{\left(1-\left|\zeta_{k_{l}, j_{l}}\right|\right)^{2}}{4} \leq\left|\Delta_{k_{l}, j_{l}}\right| \leq 4\left(1-\left|\zeta_{k_{l}, j_{l}}\right|\right)^{2}, \quad 1 \leq j \leq n
\end{gathered}
$$

and therefore, by Lemma 4 in [2], we have

$$
\begin{aligned}
I^{p} & \leq C_{1}(\omega)\|g\|_{B_{\widetilde{\omega}}}^{p} \sum_{|k|=0}^{\infty} \sum_{j=-2^{k}}^{2^{k}-1} \max _{\zeta \in \bar{\Delta}_{k, j}}|f(\zeta)|^{p} \omega\left(\left|\Delta_{k, j}\right|^{1 / 2}\right) \\
& \leq C_{1}(\omega)\|g\|_{B_{\tilde{\omega}}}^{p}\|f\|_{A^{p}(\omega)}^{p} .
\end{aligned}
$$


Using the Cauchy formula, it is easy to show that $\Phi\left(e_{z}\right)=g(z)$. Thus, the inequality $C_{1}(\omega)\|\Phi\| \leq\|g\|_{\widetilde{B}_{\widetilde{\omega}}}$ is also true.

\section{References}

[1] Z. Zhou, The essential norm of composition operator between generalized bloch spaces in polydiscs and its applications, Preprint, arXiv: math. Fa/0503723v3, 2005.

[2] F. Shamoyan, Diagonal mappings and questions of presentation in anisotropic spaces in Polydisk [in Russian], Sib. Mat. Journ., 3 (2) (1990), 197-215.

[3] K. Zhu, Bloch-type spaces of analytic functions, Rocky Mountain J. Math., 23 (1993), 1143-1177.

[4] Functions of regular variation [in Russian], Nauka, Moscow.

[5] M. M. Djrbashian, On the representation problem of analytic functions, Soobsh. Inst. Matem. Mekh. Akad. Nauk Arm SSR, 2 (1948), 3-40.

[6] N. Zorboska, Toeplitz operators on the Bloch spaces, ISAAC, 2003, York University Toronto, ON, Canada.

[7] A. Harutyunyan, Toeplitz operators and division theorems in anisotropic spaces of holomorphic functions in the polydisk, Complex Variables, 48 (4) (2003), 347-363.

Department of Mathematics Yerevan State University

Alex Manoogian St. 1

375049 Yerevan

(E-mail : anahit@ysu.am)

(Received : October 2005) 


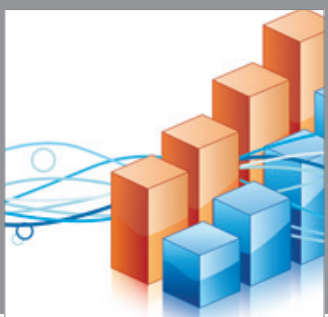

Advances in

Operations Research

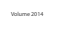

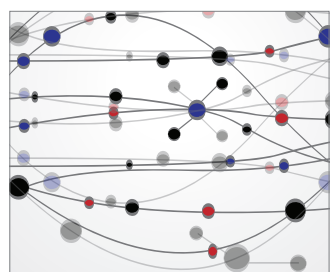

\section{The Scientific} World Journal
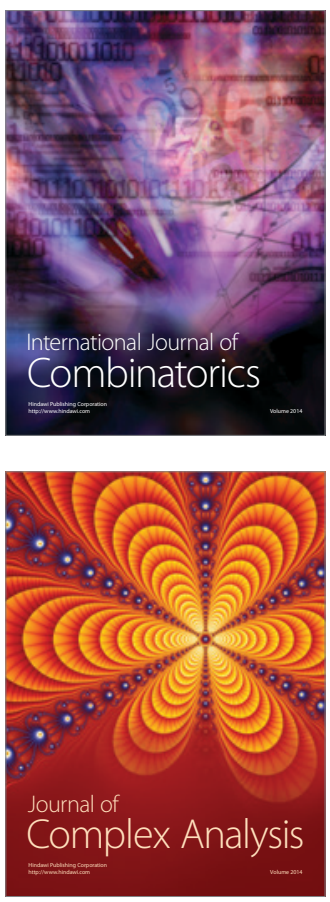

International Journal of

Mathematics and

Mathematical

Sciences
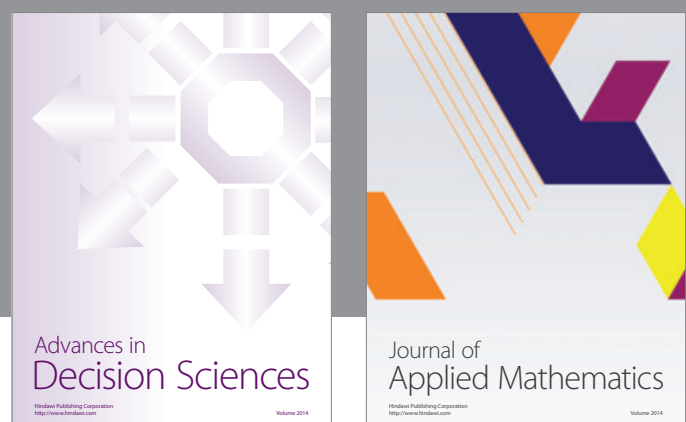

Journal of

Applied Mathematics
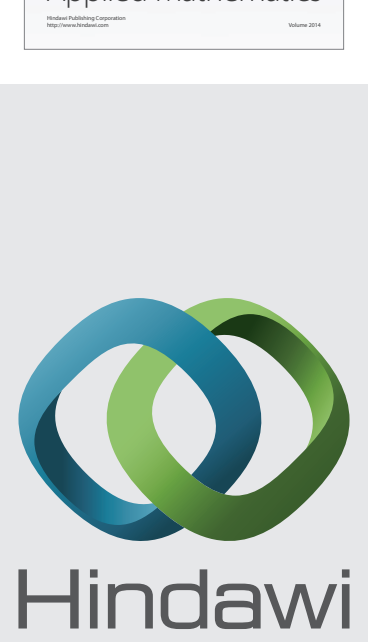

Submit your manuscripts at http://www.hindawi.com
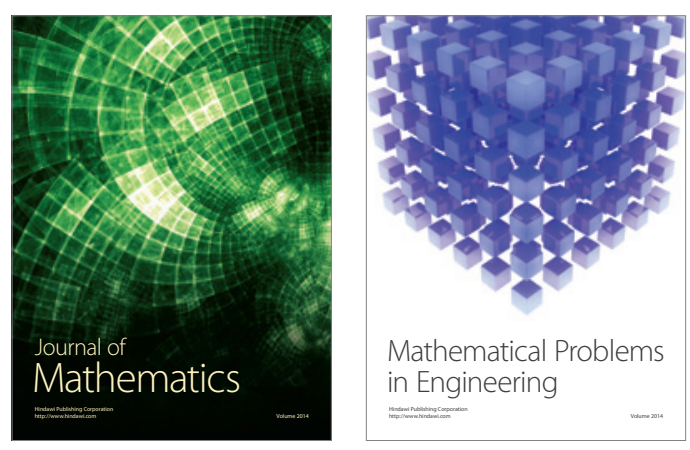

Mathematical Problems in Engineering
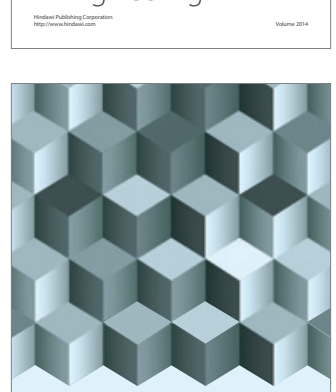

Journal of

Function Spaces
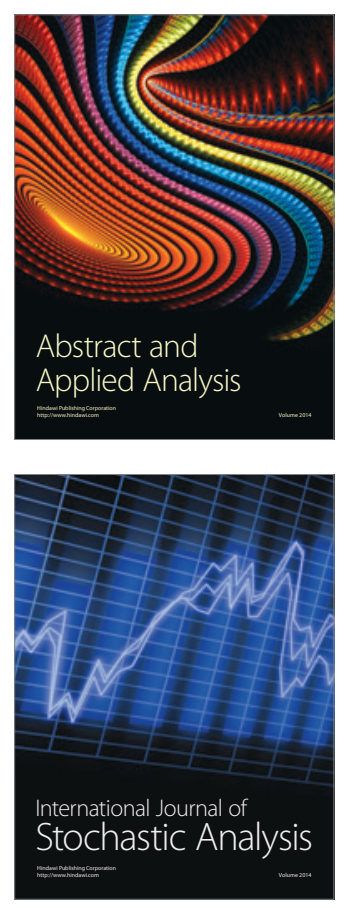

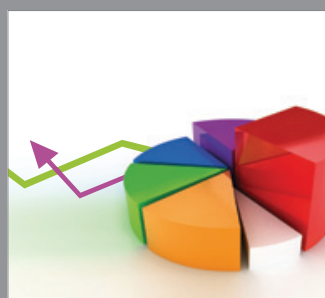

ournal of

Probability and Statistics

Promensencen
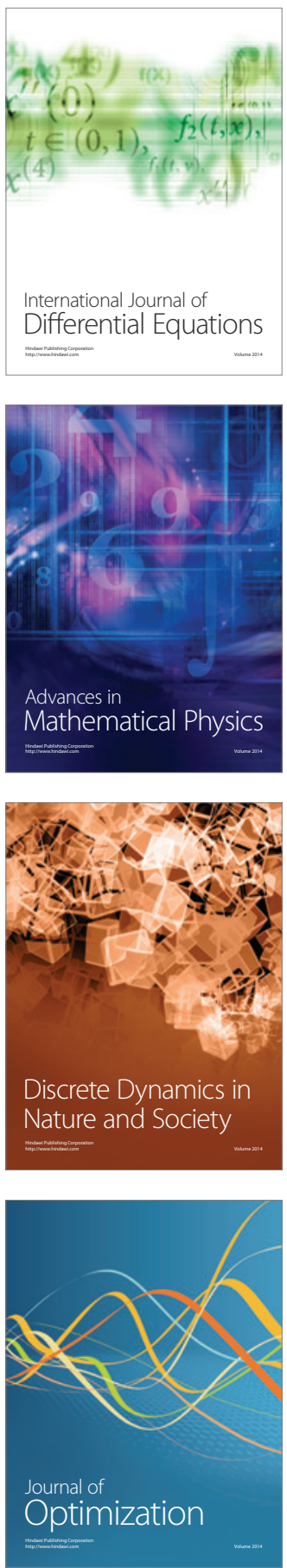